\title{
CT-MRI LI-RADS v2017: A Comprehensive Guide for Beginners
}

\author{
Francesca Patella ${ }^{1}$, Filippo Pesapane ${ }^{* 1}$, Enrico Maria Fumarola ${ }^{1}$, Ilaria Emili ${ }^{1}$, \\ Riccardo Spairani ${ }^{1}$, Salvatore Alessio Angileri ${ }^{2}$, Silvia Tresoldi ${ }^{2}$, Giuseppe Franceschelli ${ }^{2}$ \\ and Gianpaolo Carrafiello ${ }^{2}$
}

${ }^{1}$ Postgraduation School in Radiodiagnostics, Università degli Studi di Milano, Milan, Italy; ${ }^{2}$ Department of Health Sciences, Diagnostic and Interventional Radiology, ASST Santi Paolo e Carlo, San Paolo Hospital, Milan, Italy

\begin{abstract}
Hepatocellular carcinoma ( $\mathrm{HCC}$ ) is the most common primary liver malignancy and the second leading cause of cancerrelated deceases worldwide. Early diagnosis is essential for correct management and improvement of prognosis. Proposed for the first time in 2011 and updated for the last time in 2017, the Liver Imaging-Reporting and Data System (LI-RADS) is a comprehensive system for standardized interpretation and reporting of computed tomography (CT) and magnetic resonance imaging (MRI) liver examinations, endorsed by the American College of Radiology to achieve congruence with HCC diagnostic criteria in at-risk populations. Understanding its algorithm is fundamental to correctly apply LI-RADS in clinical practice. In this pictorial review, we provide a guide for beginners, explaining LI-RADS indications, describing major and ancillary features and eventually elucidating the diagnostic algorithm with the use of some clinical examples.

Citation of this article: Patella F, Pesapane F, Fumarola EM, Emili I, Spairani R, Angileri SA, et al. CT-MRI LI-RADS v2017: A comprehensive guide for beginners. J Clin Transl Hepatol 2018;6(2):222-236. doi: 10.14218/JCTH.2017.00062.
\end{abstract}

\section{Introduction}

Responsible for approximatively 750,000 deaths worldwide, hepatocellular carcinoma ( $\mathrm{HCC}$ ) is the most common primary liver malignancy and the second leading cause of cancer-related death. ${ }^{1}$ It is universally acknowledged that the etiology of $\mathrm{HCC}$ is strictly related to cirrhosis, with almost $80 \%$ of HCC cases arising in cirrhotic liver ${ }^{2}$ and with an annual incidence ranging from $2 \%$ to $8 \%{ }^{3}$ However, even if less frequently, HCC can also develop in noncirrhotic livers, when affected by chronic inflammation due to viral infections (especially linked to hepatitis $B$ virus (HBV) and hepatitis $C$ virus (HCV) $)^{2,4}$ or

Keywords: LI-RADS; Hepatocellular carcinoma (HCC); Cirrhotic livers; Radiology. Abbreviations: ADC, apparent diffusion coefficient; APHE, arterial phase hyperenhancement; CMOAT, canalicular multispecific organic anion transporter; CT, computed tomography; DWI, diffusion-weighted imaging; ECA, extracellular contrast agent; $\mathrm{FNH}$, focal nodular hyperplasia; HBA, hepatobiliary contrast agent; HBV, hepatitis $B$ virus; $\mathrm{HCA}$, hepatocellular adenoma; $\mathrm{HCC}$, hepatocellular carcinoma; $\mathrm{HCV}$ hepatitis $\mathrm{C}$ virus; ICC, intrahepatic cholangiocarcinoma; LI-RADS, Liver ImagingReporting and Data System; MRI, magnetic resonance imaging; OATP, organic anionic transporting polypeptides; WO, wash-out.

Received: 30 September 2017; Revised: 2 December 2017; Accepted: 5 December 2017

* Correspondence to: Filippo Pesapane, Postgraduation School in Radiodiagnostics, Università degli Studi di Milano, Via Festa del Perdono 7, Milan 20122, Italy. Tel: +39-13012751123; Fax: +39-2-50323393; E-mail: filippo.pesapane@unimi.it nonalcoholic steatohepatitis. ${ }^{5}$ Interestingly, the same environment also predisposes to the onset of intrahepatic cholangiocarcinoma (ICC), ${ }^{6,7}$ while it reduces the risk of liver metastases. ${ }^{8}$

The most important changes arising during HCC carcinogenesis and the corresponding radiological signs are summarized in Table $1.9,10$ The prognosis of HCC is largely influenced by the disease stage at the time of detection, as it has been assessed that early-stage $\mathrm{HCC}$ can be managed with a number of potentially curative treatments. ${ }^{11,12}$ Therefore, early diagnosis of HCC is critical to improve the survival of affected patients. ${ }^{10}$ Recommended by both the European Association for the Study of the Liver and the American Association for the Study Liver Diseases, CT and MRI are currently recognized as the most useful noninvasive tools for active surveillance of $\mathrm{HCC}$ in at-risk populations. ${ }^{13-15}$

In the past, several clinical practice guidelines have been suggested by organizations all over the world, in the attempt to standardize imaging-based criteria for HCC diagnosis. ${ }^{16}$ Nevertheless, these guidelines have some limitations, such as the inconsistency between different systems in definition of pathological features, the extent of the indeterminate category and the lack of indications for differential diagnosis from other malignancies or for characterization of macrovascular invasion. ${ }^{17,18}$ Proposed for the first time in 2011 and then updated in 2013, 2014 and 2017, the Liver Imaging-Reporting and Data System (LI-RADS) is a comprehensive system for standardized interpretation and reporting of CT and MR liver examinations, endorsed by the American College of Radiology to overcome the mentioned limits and achieve congruence with HCC diagnostic criteria in at-risk populations. ${ }^{17,18}$

Describing some major and ancillary imaging features to apply through a precise algorithm, LI-RADS classifies CT or MRI observations into five categories, from LR-1 to LR-5, corresponding to a crescent probability of HCC, where LR-1 and LR- 5 represent respectively the $0 \%$ and $100 \%$ probability of HCC (Table 2). ${ }^{19,20}$ Thus, the system provides three labels (LR-2, LR-3 and LR-4) to differentiate uncertain observations, responding to the need of narrowing the broad indeterminate category, that used to result from the dichotomic approach of "definite versus nondefinite HCC". ${ }^{18}$ Importantly, LI-RADS defines a further category of LR-TIV (LR-tumor in vein) for lesions associated with macrovascular invasion, moving towards the TNM staging system, which requires the delineation of the vascular involvement to plan the treatment strategy. ${ }^{18,20}$ Moreover, malignancies other than HCC can be classified under the label LR-M using LI-RADS-specific guidelines for differential diagnosis. ${ }^{20}$ Eventually, LI-RADS provides a structured reporting template, recommendations on optimal 


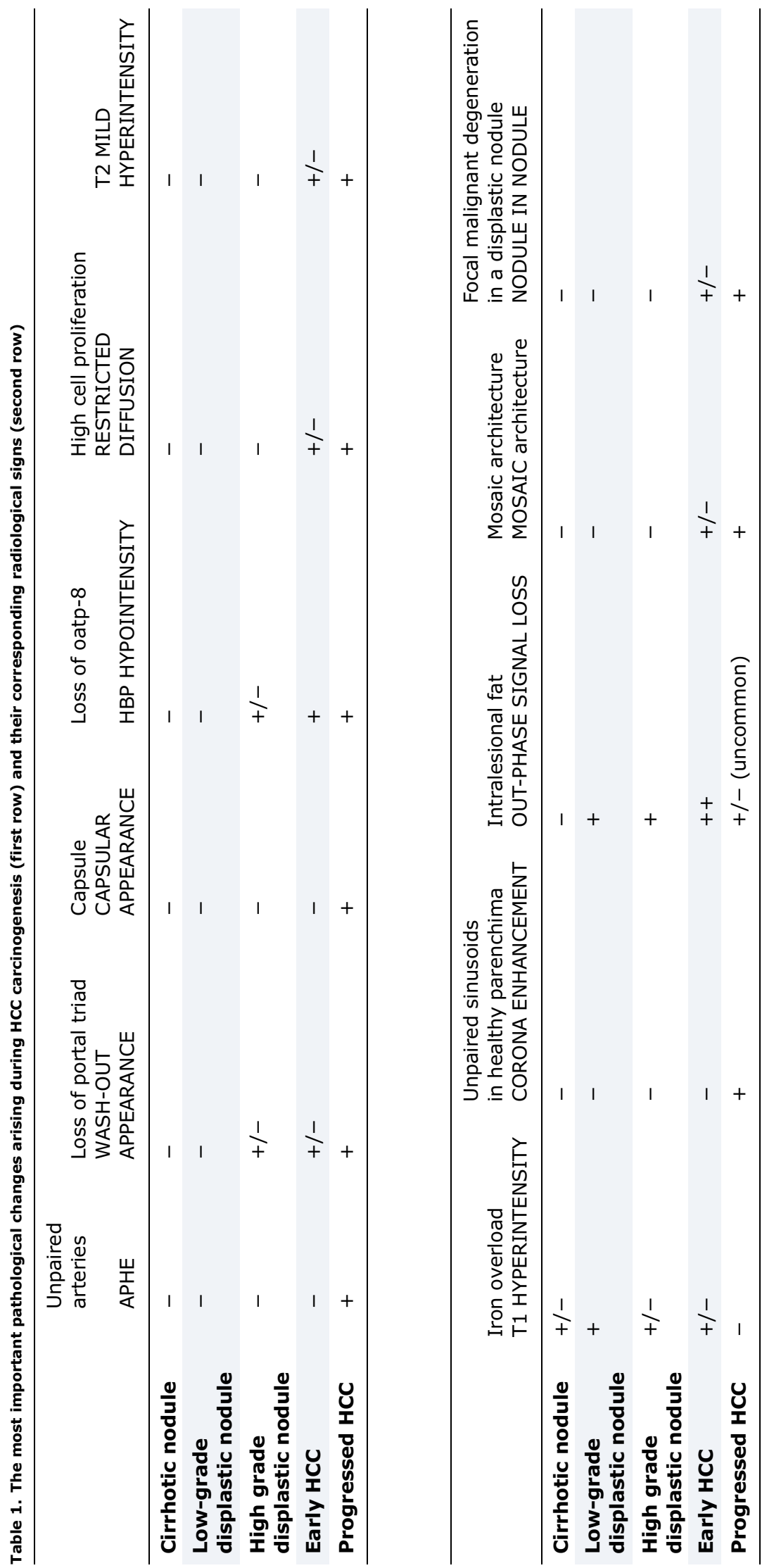


Table 2. LI-RADS diagnostic categories (on the left) and examples of LR-1 and LR-2 entities (on the right). From the American College of Radiology - Liver imaging reporting and data system version (LI-RADS $®$ ) $v 2017$

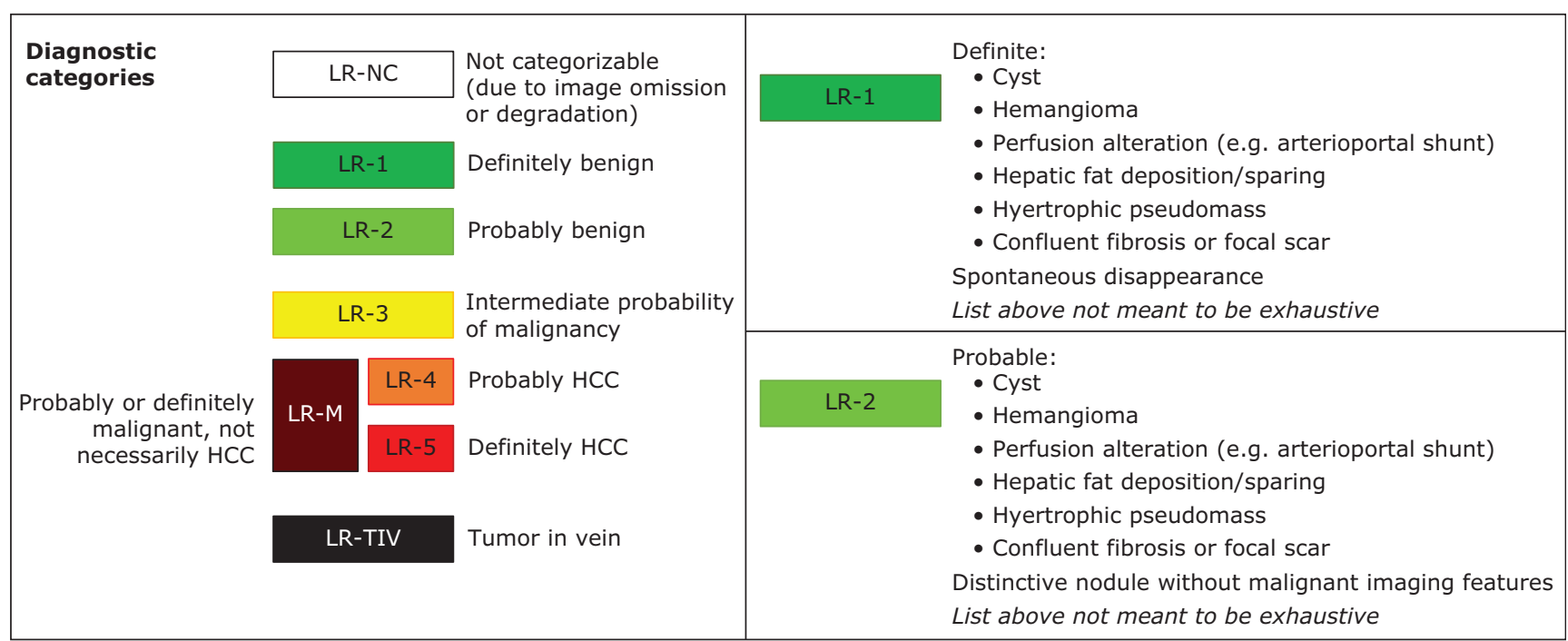

imaging protocol, management suggestions and, in the last update (v2017), criteria for CT/MRI treatment response assessment. ${ }^{20}$

In this pictorial review, we aim to explain the LI-RADS algorithm, summarizing the major and ancillary features, in order to supply an educational guide for practical use for CT and MRI diagnosis of HCC.

\section{Indications}

LI-RADS provides a CT/MRI diagnostic algorithm that can be applied only in a high-risk population for $\mathrm{HCC}^{20}$ Namely, the last version defines as high-risk patients those affected by cirrhosis, chronic hepatitis B without cirrhosis or by a prior HCC, including adult liver transplant candidates and recipients posttransplantation. ${ }^{20}$ Conversely, LI-RADS cannot be used in the absence of the above risk factors or in a pediatric population, even when affected by cirrhosis due to congenital hepatic fibrosis, since its use has not been validated for patients less than 18-years-old. ${ }^{19}$

Interestingly, LI-RADS excludes patients with cirrhosis due to vascular disorders, such as hereditary hemorrhagic telangiectasia, Budd-Chiari syndrome, chronic portal vein occlusion, cardiac congestion, or diffuse nodular regenerative hyperplasia, ${ }^{20}$ since these conditions represent an environment predisposing to the development of benign arterialized nodules that may mimic HCC, leading to misdiagnosis. ${ }^{19}$ Furthermore, LI-RADS v2017 recommends application of the algorithm only for multiphase imaging examinations either performed with CT or MRI and with extracellular contrast agents (ECAs) or MRI with hepatobiliary contrast agents (HBAs). ${ }^{20}$ Contrarily, singlephase or double-phase CT-MRI cannot be considered adequate for the employ of LI-RADS algorithm. ${ }^{20}$

Eventually, LI-RADS algorithm should be proscribed for observations that are path-proven malignancies or pathproven benign lesions of nonhepatocellular origin, such as hemangiomas.

\section{Major and ancillary features}

LI-RADS defines criteria that should be used to classify a liver observation. Here, the term "observation" refers to a focal area with imaging features that differ from those of adjacent liver parenchyma, and replaces the terms "lesion" or "nodule" since it also includes "pseudolesions", such as artifacts or perfusion alterations. ${ }^{18}$ The imaging features selected by LI-RADS are divided into two main groups, according to their role in the diagnostic algorithm: the major and ancillary features. ${ }^{20}$

\section{Major features}

LI-RADS provides four major features-arterial phase hyperenhancement (APHE), wash-out (WO) appearance, capsule appearance, and threshold growth. These features are valid both for CT and MRI, since they can be detected by any dynamic contrast-enhanced examination and performed with administration of either extracellular or hepatospecific contrast agents, providing that it includes three dynamic phases: the arterial, portal and delayed phase. ${ }^{20}$ Although the individual features are nonspecific, the combination of them is highly specific for $\mathrm{HCC}$ in at-risk populations, ${ }^{21-23}$ reaching approximately $100 \%$ specificity for $10-19 \mathrm{~mm}$ observations featured by visibility as a distinctive nodule at former surveillance ultrasound, APHE and WO appearance. ${ }^{9,21}$ The main limitation of employment of major features for diagnosis and staging of HCC is the low per-lesion sensitivity, since most (e.g., the APHE and the capsule appearance) are typical of progressed HCC and barely useful in early HCC detection. ${ }^{9,10}$

\section{APHE}

LI-RADS defines APHE as a nonrim-like enhancement in the arterial phase that is unequivocally greater in attenuation or intensity in whole or in part than the liver (Figs. $1 \mathrm{~A}$ and $2 \mathrm{~A}$, 2C). ${ }^{20}$ It must be noticed that the expression "arterial phase" refers to the "hepatic arterial phase", which is featured by 
simultaneous full enhancement of the hepatic artery and branches and by the absence of hepatic vein enhancement. ${ }^{20}$ Moreover, LI-RADS distinguishes two subtypes-the early and the late arterial phase, respectively characterized by the absence or the presence of portal vein enhancement, with recommended use of the latter since APHE is often stronger or even detectable only in this phase. ${ }^{20}$ Also called "wash-in" or "arterial hypervascularity", APHE is a dynamic profile correlated to neoangiogenesis with formation of impaired arteries that occurs late during hepatocarcinogenesis. ${ }^{10,24}$

Being a distinctive feature of progressed HCC, APHE has a prominent role in the LI-RADS algorithm. ${ }^{18,20}$ However, APHE is not completely specific, since it can also be observed in benign perfusion alterations, small hemangiomas, small focal nodular hyperplasia-like lesions, ${ }^{25,26}$ in some atypical cases of focal or confluent fibrosis and in some atypical cirrhotic and dysplastic nodules or small hypervascular metastases such as neuroendocrine tumors. ${ }^{9,16}$ In addition, in patients with cirrhosis or chronic hepatitis, small vascular pseudolesions attributable to arteroportal shunts are particularly common, and the large majority of focal areas of enhancement seen only in the arterial phase and measuring $<2 \mathrm{~cm}$ are nonneoplastic, ${ }^{27}$ especially those that are wedge-shaped and subcapsular. ${ }^{28}$ Furthermore, APHE sensitivity decreases with the diameter of the observations, dropping from $100 \%$ to $32 \%$ for observations, respectively, for sizes of $>20 \mathrm{~mm}$ and $<10 \mathrm{~mm}$, even using MRI. ${ }^{29}$
WO appearance

WO appearance is defined as a nonperipheral, visuallyassessed, temporal reduction in enhancement in whole or in part compared to the surrounding liver parenchyma from the earlier to later phase, resulting in hypoenhancement in the extracellular phase (Figs. 1B, 2B, 2D and 3B). ${ }^{20}$ Since this pattern can be more conspicuous or even depictable only in the delayed phase, ${ }^{30,31}$ the LI-RADS recommends to state WO appearance evaluating of both portal venous and delayed phases when administrating ECAs or gabobenate. ${ }^{20}$ Conversely, in case of employment of gadodexate, only portal venous phase should be considered and any hypointensity in the transitional phase (which is not a real delayed phase) should be reported as an ancillary feature and not as a WO appearance. ${ }^{20}$

Importantly, LI-RADS advocates the expression "WO appearance" instead of the simple WO, since the mechanisms underlying this pattern in HCC are not fully understood. Indeed, several concurrent factors have been hypothesized, such as early venous drainage of contrast material from the tumor, progressive enhancement of background liver, reduced intranodular portal venous blood supply, tumoral hypercellularity, and intrinsic hypoattenuation/hypointensity. ${ }^{32}$ Like APHE, WO appearance by itself is not specific for HCC, as this feature may be observed in cirrhotic and dysplastic nodules, in metastasis and in hepatocellular adenoma, even if the last two conditions are both rare in the HCC at-risk population. $8,10,33$

\begin{tabular}{|c|c|c|c|c|c|c|}
\hline \multirow{2}{*}{\multicolumn{2}{|c|}{$\begin{array}{l}\text { Arterial phase hyperenhancement (APHE) } \\
\text { Observation size }(\mathrm{mm})\end{array}$}} & \multicolumn{2}{|c|}{ No APHE } & \multicolumn{3}{|c|}{ APHE (not rim) } \\
\hline & & $<20$ & $\geq 20$ & $<10$ & $10-19$ & $\geq 20$ \\
\hline \multirow{3}{*}{$\begin{array}{l}\text { Count major features: } \\
\text { - "Washout" (not peripheral) } \\
\text { - Enhancing "capsule" } \\
\text { - Threshold growth }\end{array}$} & None & LR-3 & LR-3 & LR-3 & LR-3 & LR-4 \\
\hline & One & LR-3 & LR-4 & LR-4 & LR-4 LF & LR-5 \\
\hline & $\geq$ Two & LR-4 & LR-4 & LR-4 & LR-5 & LR-5 \\
\hline
\end{tabular}

\section{LR-4 Observations in this cell are categorized LR-4, except:}

- LR-5g, if $\geq 50 \%$ diameter increase in $<6$ months (equivalent to OPTN $5 A-g$ )

- LR-5us, if "washout" and visibility at screening ultrasound (per AASLD HCC criteria)

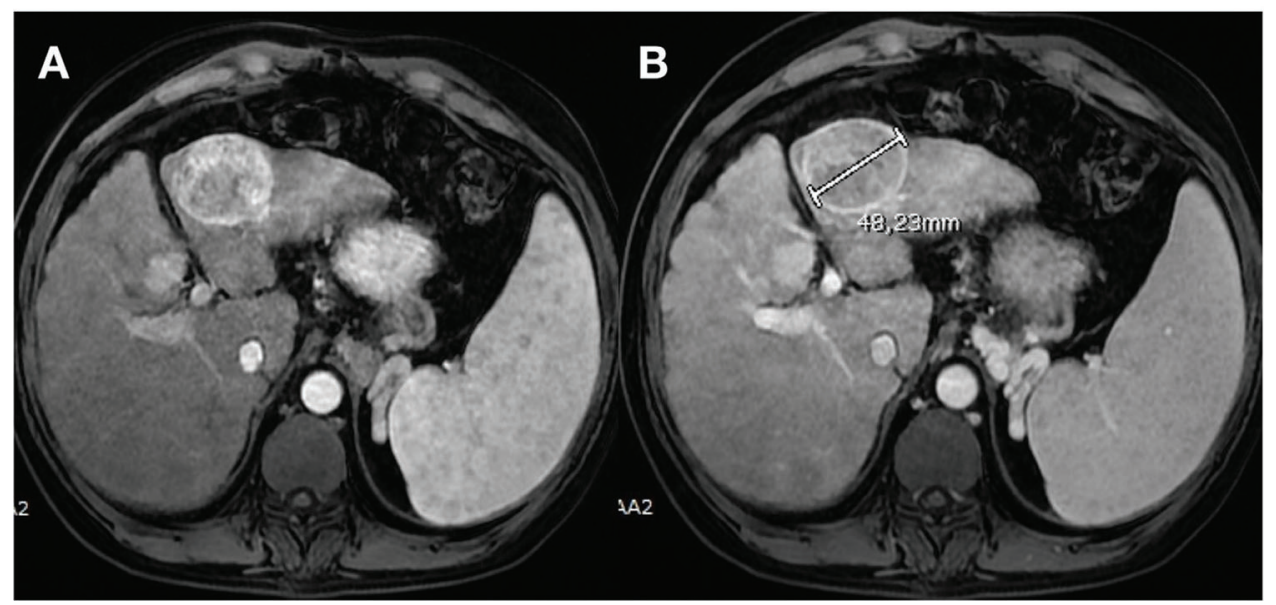

Fig. 1. LI-RADS algorithm for application of major criteria (on the top). From the American College of Radiology - Liver imaging reporting and data system version

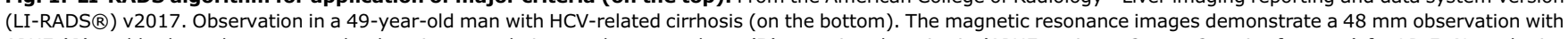
APHE (A) and both wash-out out and enhancing capsule in portal venous phase (B), meeting the criteria (APHE + size $>2 \mathrm{~cm}+2 \mathrm{major}$ features) for LR-5. Note the inhomogeneous structure of the observation reflecting a "mosaic" architecture. 


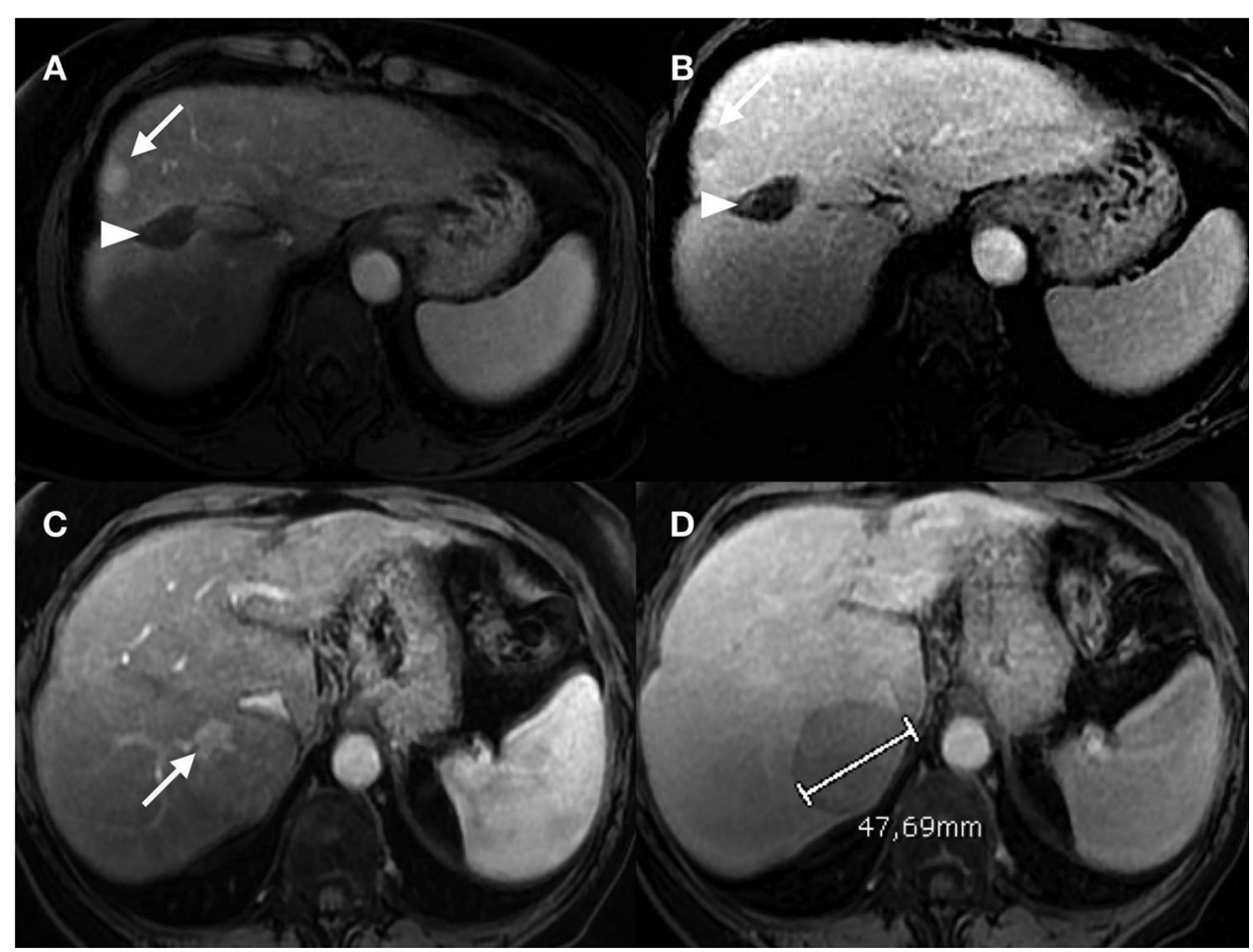

Fig. 2. Particular cases of LR-5 on MRI. (A, B) 14-mm observation in a 73-year-old woman with chronic hepatitis B, previously treated for HCC. (A) Arterial phase demonstrating APHE (arrow). (B) Portal venous phase demonstrating "wash-out" (arrow) and no enhancing capsule or threshold growth. Since the observation has APHE, $10<\mathrm{d}<20 \mathrm{~mm}$ and one major feature, the final category is LR-4/5. The nodule was reclassified the same day as a LR-5us, as it was visible at ultrasound examination. The hypointense triangular area (arrowhead) has to be correlated to previous treatments. (C, D) 48-mm nodule (arrow) in a 73-old-woman with hepatitis C cirrhosis. (C) Arterial phase demonstrating a focal APHE in a bigger surrounding nodule (arrow). (D) Portal venous phase showing "wash-out". Thus, the observation is featured APHE + size $>2 \mathrm{~cm}$ plus one major feature. The final category is LR-5. Note, in this case the focal APHE is inside a larger nonenhancing nodule, reflecting the "nodule-in-nodule" architecture.

Additionally, focal areas of parenchymal distortion and enhancing fibrosis may create a perception of WO, named "pseudoWO". ${ }^{34}$

\section{Enhancing "capsule"}

LI-RADS defines an enhancing "capsule" as a smooth, homogenous, sharp border surrounding entirely or partly an observation, unequivocally thicker or more conspicuous than fibrotic tissue around neighboring cirrhotic nodules and visible as a rim featured by progressive enhancement from early to later phases (Fig. 1B). ${ }^{20}$ This characteristic dynamic pattern has been attributed to slow flow within intracapsular vessels, as well as contrast agent retention within the extravascular connective tissue of the capsule. ${ }^{35}$ Nevertheless, it should remarked that LI-RADS reports the term "capsule" with quotation marks, since the rim of enhancement does not necessarily correlate with the presence of a true tumor capsule at pathology ${ }^{19}$ but it may represent a pseudocapsule corresponding to fibrous tissue and dilated sinusoids around a nodule. ${ }^{36}$ In both cases, the enhancing "capsule" is a specific feature of progressed $\mathrm{HCC}^{23,34-37}$ since it is normally absent in precursor nodules (cirrhotic nodules and dysplastic nodules) and nonHCC tumors. ${ }^{23,34,37}$

Indeed, this major feature has shown itself to be an important predictor of $\mathrm{HCC}$, permitting definitive diagnosis of $\mathrm{HCC}$ when associated with APHE and an observation size of $2 \mathrm{~cm}$ or larger, regardless of the presence of WO appearance. ${ }^{34,38} \mathrm{~A}$ potential pitfall in applying this feature is that some small ICCs show peripheral enhancement in all phases, ${ }^{39}$ which may be misinterpreted as a "capsule"; a discriminating characteristic is that the peripheral enhancement in ICC tends to peak in the arterial phase and diminish in later phases, rather than progress. Another possible snare is that fibrous tissue surrounding cirrhotic nodules and dysplastic nodules may enhance on delayed phase images, generating the perception of a "capsule". ${ }^{34}$ Thus, LI-RADS specifies the term "unequivocally" in the definition of the enhancing "capsule", in order to prevent an inappropriate application of this feature. ${ }^{20}$

Moreover, importantly, LI-RADS v2017 adds the adjective "enhancing" to "capsule" in the algorithm table as well, to highlight the distinction from "nonenhancing capsule", which is a peripheral rim visible in other MRI sequences such as T2weighted or the hepatobiliary phase (HBP) that should be considered just as an ancillary feature favoring HCC. ${ }^{20}$

\section{Threshold growth}

The definition of HCC growth is an object of debate because growth has been variably defined in the various studies that have contributed to consensus criteria. ${ }^{18}$ LI-RADS defines growth as an unequivocal increase in the diameter of an observation, measured on examinations performed on different dates, which is not attributable to artifact, differences in technique between the two examinations, or measurement error. ${ }^{20}$ Growth can be considered as a major feature of HCC whenever it achieves a threshold defined as an increase in maximum diameter of at least $50 \%$ within 6 months or at least $100 \%$ 
One or more ancillary features favoring malignancy: upgrade by 1 category up to LR-4 (Absence of these ancillary features should not be used to downgrade)

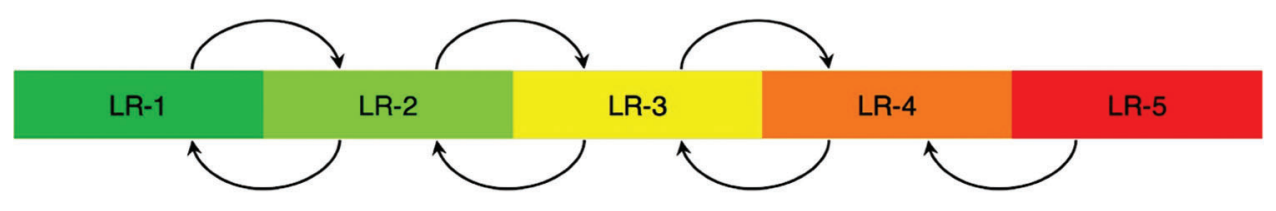

One or more ancillary features favoring benignity: downgrade by 1 category (Absence of these ancillary features should not be used to upgrade)

If there are conflicting AFs (i.e., one or more favoring malignancy and one or more favoring benignity): Do not adjust category

Ancillary features cannot be be used to upgrade to LR-5

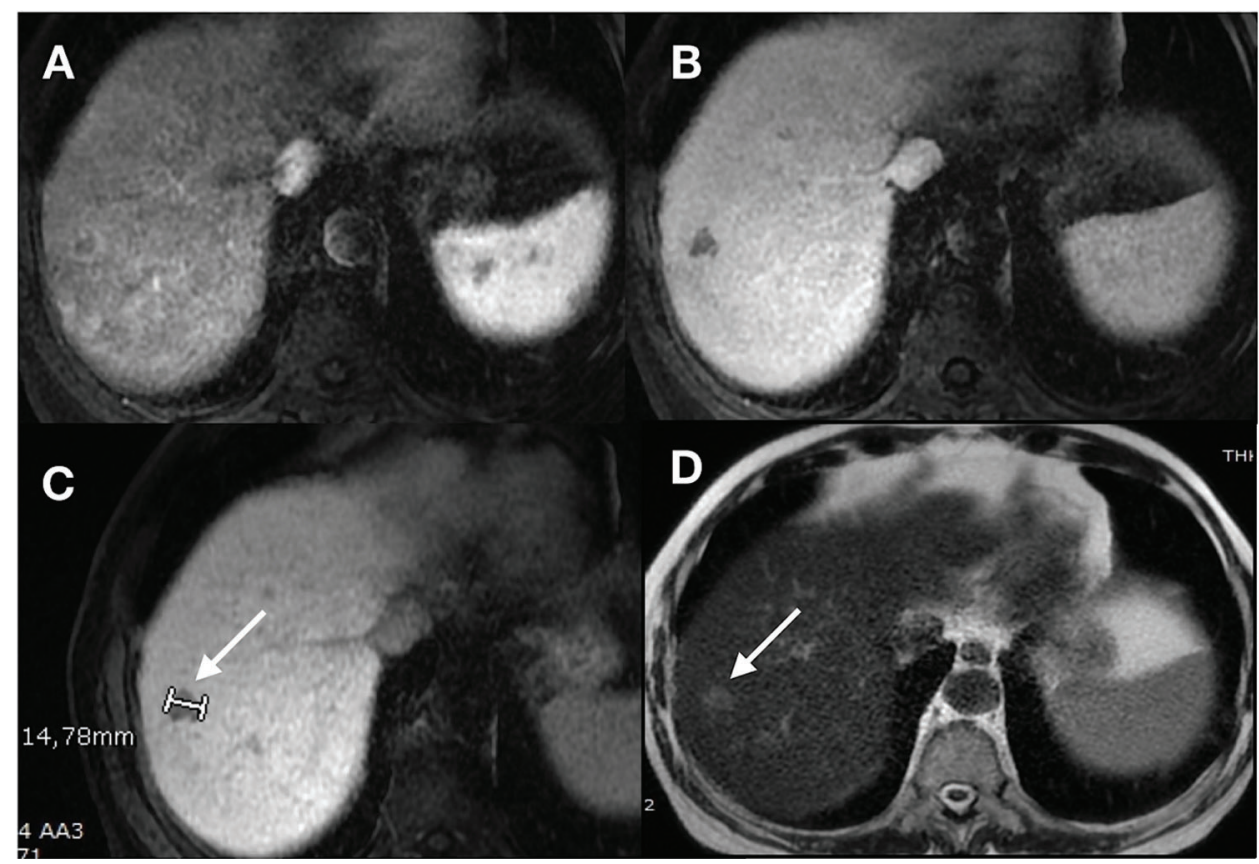

Fig. 3. LI-RADS algorithm for application of ancillary criteria (on the top). From the American College of Radiology - Liver imaging reporting and data system version (LI-RADS $®$ ) v2017. Magnetic resonance images showing a 15-mm nodule (arrow) in a 77-year-old man with hepatitis C-related cirrhosis (on the bottom). (A) Arterial phase does not demonstrate a clear APHE. (B) Portal venous phase shows "wash-out", a major feature for HCC. Since the observation has no APHE, it is <20 mm and has only one major feature favoring HCC, it was initially classified as LR-3 according to the left part of the table algorithm. However, the observation was hypointense in the hepatobiliary phase (C) and slightly hyperintense in T2W images (D). So, using these two ancillary features favoring malignancy, we could upgrade the LI-RADS score to the final category of LR-4, probably a HCC. The patient underwent a biopsy which confirmed the diagnosis.

diameter increase per year. ${ }^{20}$ Despite HCC growth rate varies widely, secondarily to the size or histologic grade of the tumors, LI-RADS decided to fix a threshold in order to decrease the likelihood of a false-positive diagnosis caused by slow-growing benign entities, including some dysplastic nodules. ${ }^{18}$

Interestingly, new lesions measuring at least $1 \mathrm{~cm}$ are also given credit for threshold growth if they were not visible at a CT or MRI that had been performed less than 1 year before. ${ }^{16,20}$ A diameter increase of less than the threshold growth is an ancillary feature that favors HCC. ${ }^{18,20}$ In this case, LI-RADS does not stipulate a minimum increase in diameter required for use as an ancillary feature. ${ }^{20}$ Since it is a major criterion for the diagnosis of HCC, radiologists should exercise judgment in the application of the threshold growth feature, which refers only to an unequivocal increase in the diameter of an observation. ${ }^{18,20}$ Moreover, diameter increase should be assessed on images in the same plane and, if possible, acquired in the same phase or sequence. ${ }^{20}$

Because the difficulty in differentiating nodules from surrounding peritumoral enhancement can lead to overestimation of tumor size, LI-RADS recommends measurement on phases that are relatively constant over time (e.g., venous or precontrast phases). ${ }^{20}$

\section{Ancillary features}

Although their presence is an accurate positive indicator of HCC, major features were found to underestimate its occurrence by $40-60 \%$ of cases according to the size of the 
lesion. ${ }^{14,34,40}$ Therefore, in addition to these standard diagnosis methods, others have been investigated that may be employed for enhanced accuracy. ${ }^{17,41}$ LI-RADS defines these additional signs as "ancillary features", classifying them into two groups. The first group comprises those signs favoring malignancy, but which are not specific of HCC. The second group includes those markers that characterize HCC especially. ${ }^{20}$ The list of all LI-RADS ancillary features is reported in Table $3 .^{20}$

Below, we discuss some of the main ancillary features favoring malignancy and HCC in particular.

\section{Ancillary features favoring malignancy, but not HCC in particular}

HBP hypointensity

Hepatocellular adenomas (HCAs) are paramagnetic gadolinium chelates, determining shortening of the $\mathrm{T} 1$ relaxation time, that, compared to conventional extracellular gadolinium-based contrast agents, are able to provide similar arterial and portal venous phases, but because of their hepatocyte specific uptake they also enable an HBP. ${ }^{42,43}$ Gadobenate dimeglumine and gadoxetic acid are the two liver-specific MRI contrast agents used in clinical practice. ${ }^{9}$ After the dynamic phase, a specific transporter receptor called the organic anion transporter (OATP), which is part of the biliary transport system, carries these agents into hepatocytes. Later, the agents are excreted into bile canaliculi via the multidrug resistance-associated protein (also known as the canalicular multispecific organic anion transporter; cMOAT). ${ }^{44}$

Robust evidence have shown that OATP expression declines during hepatocarcinogenesis, so the evaluation of signal intensity in the hepatobiliary phase can help to identify and characterize hepatocellular nodules in the cirrhotic liver. ${ }^{9}$ Moreover, hepatobiliary agents permit the assessment of hepatocellular function as well, based on signal intensity of the liver in the HBP, which tends to drop in patients with impaired liver function. ${ }^{9}$

Ideally, $50 \%$ of the injected dose of gadoxetate disodium is eliminated in the bile, with a peak of parenchymal enhancement that occurs at approximately $20 \mathrm{~min}$ after the injection of the contrast media so that a 20-min delay acquisition usually results in an adequate HBP. ${ }^{45}$ Gadobenate dimeglumine has lower biliary excretion (2 4\%), so an adequate HBP can be reached usually in $1 \mathrm{~h}$ in normal patients, but a delay of $1-3 \mathrm{~h}$ is necessary in patients with impaired liver function or a severe cirrhosis. ${ }^{45}$ LI-RADS defines the HBP hypointensity as an intensity in the HBP that is unequivocally less, in whole or in part, than liver. ${ }^{20}$ This feature constitutes a very sensitive sign, since it can be seen in $79-99 \%$ of $\mathrm{HCCs}^{46}$ including well-differentiated early HCCs (Fig. 3C). ${ }^{47}$

What pathological data suggest is that, in cirrhotic liver, OATP expression is still preserved in dysplastic nodules (which in HBP typically have similar signal intensity to surrounding liver parenchyma); it starts to decline just after malignant transformation, before the arterialization of the nodule is realized..$^{9,10}$ This a key point, because it means that HBP hypointensity allows the detection of HCC, even before the onset of APHE. ${ }^{9,10}$ Given that approximately $20-40 \%$ of lesions smaller than $20 \mathrm{~mm}$ in diameter do not show a clear enhancement in the arterial phase, the HBP represents an essential tool to increase MRI diagnostic accuracy, allowing for differential diagnosis between HCC and dysplastic nodules, even when conventional imaging fails. ${ }^{48}$

Moreover, HBP hypointensity can help to differentiate between hypervascular small HCCs and arteriovenous shunts or focal nodular hyperplasia (FNH)-like lesions, since such observations remain iso- or hyperintense in $\mathrm{HBP}$, in contrast to HCC. ${ }^{48}$ Despite HBP hypointensity is a reliable predictor of HCC in at-risk populations, ${ }^{42}$ it lacks specificity since it can also be observed in some atypical dysplastic nodules, in all other liver malignancies (metastases included) and in some benign entities like hemangiomas. ${ }^{49}$ Furthermore, radiologists should be aware that almost $10 \%$ of well-differentiated HCC show HBP hyperintensity, instead of the expected hypointensity. ${ }^{10}$ This potential pitfall relates to the fact that the dedifferentiation process can sometimes lead to overexpression of OATP8 , even if this possibility is definitely less frequent than the underexpression. ${ }^{9}$

\section{Mild-moderate $\mathrm{T} 2$ hyperintensity}

Mild-moderate T2 hyperintensity is defined as an intensity on T2-weighted images that is mildly or moderately higher than liver and similar to or less than noniron-overloaded spleen, ${ }^{20}$ but also less than bile ducts or other simple fluid-filled

Table 3. List of LI-RADS ancillary features

Ancillary features favoring malignancy

\begin{tabular}{|c|c|c|}
\hline $\begin{array}{l}\text { Ancillary features favoring } \\
\text { malignancy malignancy in general }\end{array}$ & $\begin{array}{l}\text { Ancillary features } \\
\text { favoring HCC }\end{array}$ & $\begin{array}{l}\text { Ancillary features favoring } \\
\text { benignity }\end{array}$ \\
\hline Mild-moderate T2 hyperintensity & Corona enhancement & Homogenous marked $\mathrm{T} 2$ hyperintensity \\
\hline Restricted diffusion & Mosaic architecture & $\begin{array}{l}\text { Homogeneous marked T2 } \\
\text { or T2* hypointensity }\end{array}$ \\
\hline Lesional fat sparing & Nodule-in-nodule architecture & Undistorted vessels \\
\hline Lesional iron sparing & Intralesional fat & Parallels blood pool enhancement \\
\hline Blood products & Non enhancing capsule & Diameter reduction \\
\hline Diameter increase less than threshold growth & & Diameter stability 2 years or more \\
\hline Hepatobiliary-phase hypointensity & & Hepatobiliary-phase isointensity \\
\hline Transitional phase hypointensity & & \\
\hline
\end{tabular}


structures (Figs. 3D and 4). ${ }^{9}$ The biological basis for this feature is not well-understood.

Some authors have suggested that hyperintensity on T2-weighted imaging is consistent with the alterations of vascular profile occurring during hepatocarcinogenesis, such as sinusoid dilation ${ }^{50}$ or increased intratumoral arterial supply corresponding to decreased intratumoral portal blood supply. ${ }^{47}$ However, it is still not clear whether it's the blood itself (blood volume or blood flow amount) that directly influences the signal intensity or if it depends on other factors which are yet to be determined. ${ }^{51}$ Some other data have correlated hyperintensity of HCC on T2-weighted images with the dedifferentiation of hepatocellular nodules, since progressed HCCs often result in T2 hyperintensity, ${ }^{52}$ even if APHE is not present. ${ }^{9}$ Indeed, it has been supposed that T2 elongation in HCC nodules reflects the morphological changes along hepatocarcinogenesis, from trabecular to solid growth pattern, regardless of the presence of vascularity alterations ${ }^{53}$ that may occur late. ${ }^{54}$

Usually, in T2-weighted imaging, regenerative nodules are indistinct, dysplastic nodules, appearing as iso- or hypointense, whereas early HCC is typically isointense or mildly hyperintense. ${ }^{9}$ So, any T2 elongation in at-risk patients should be considered as highly suggestive of a malignancy. ${ }^{55}$ Evidence have shown that T2-weighted imaging is particularly valuable together with diffusion-weighted imaging (DWI) for differentiating between small atypical HCCs $(<3 \mathrm{~cm})$ and dysplastic nodules, ${ }^{56}$ as the presence of mild-moderate $\mathrm{T} 2$ hyperintensity or restricted diffusion strongly favors the diagnosis of a malignant nodule in cirrhotic patients, even if the typical feature of the arterial hyperenhancement is still missing. ${ }^{10}$

Recently, a consensus report statement confirmed the importance of mild-moderate T2 hyperintensity, recommending its use in the diagnosis and staging of HCC. ${ }^{57}$ However, since mild-moderate T2 hyperintensity can be frequently observed in ICCs and liver metastases as well, ${ }^{9}$ the LI-RADS endorses its application, but only as an ancillary feature favoring generic malignancy and not specifically HCC. ${ }^{20}$

\section{Restricted diffusion}

DWI is an MRI spin-echo planar imaging sequence, sensitized to the driven random motion of water molecules (water diffusion) by the application of gradient pulses, the entity of which is indicated by a factor known as the b-value; the higher the $b$-value, the more sensitive the sequence is to water diffusion. ${ }^{58}$ The subset of DWI is the simple assumption that water diffusion in the extracellular compartment is influenced by the cell membranes and macromolecules, ${ }^{59}$ resulting in diffusion restriction whenever cellularity increases, reducing the width of interstitial spaces. ${ }^{58}$ Importantly, water diffusion can be quantified by a mathematical index called the apparent diffusion coefficient (ADC); low ADC values mean restricted diffusion, namely hypercellularity that impedes the mobility of water molecules. ${ }^{58}$

LI-RADS allows the use of restricted diffusion, as an ancillary promalignancy feature, when the observation presents an intensity on DWI that is not attributable exclusively to T2 shine-through, is unequivocally higher than liver and/or ADC, and is unequivocally lower than liver (Fig. 5A). ${ }^{20}$ One of the benefits of DWI is the it has a higher detection rate of liver tumors than conventional unenhanced MRI imaging, ${ }^{60}$ being associated with an excellent signal-to-noise ratio with suppression of background signals from vessels and bile ducts, which increases lesion conspicuity. ${ }^{61}$

In 2009, Zech et al. ${ }^{62}$ reported a higher sensitivity for DWI compared to conventional MRI in the detection of HCC in the cirrhotic liver, and Vandecaveye et al. ${ }^{63}$ concluded that DWI provided higher sensitivity and positive predictive value for the detection of $\mathrm{HCC}<20 \mathrm{~mm}$ compared to conventional contrast-enhanced MRI. Furthermore, interestingly, there are some evidence that indicate DWI may correlate to histological grade; as HCC progresses to poorly differentiated HCC, there is increased cellular density, nuclear/cytoplasmic ratio and intracellular organelles, thickened cellular plates and shrinkage of the extra- and intra-cellular spaces, ${ }^{64}$ which may lead to restricted diffusion and lower ADC values. ${ }^{65}$ However, there is no consensus about the results of these techniques so far. ${ }^{66-69}$

The main limitation of the use of DWI solely for assessing hepatic lesions is that there is a considerable overlap between benign and malignant lesions and normal liver tissue. ${ }^{70,71}$ In addition, it must be noticed that DWI images are very sensitive to artifacts for liver motion due to respiration and artifacts in the left lobe derived from the heart beating. ${ }^{72,73}$ Therefore, DWI should be used in conjunction to conventional imaging, and researchers should make every effort to improve accuracy and reproducibility of DWI, standardizing

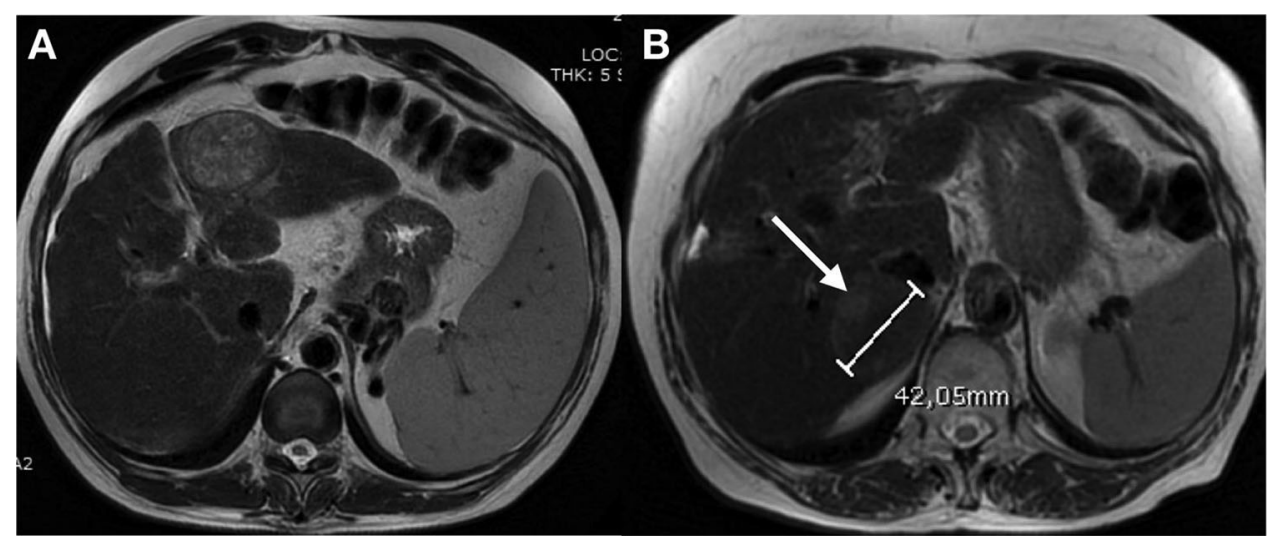

Fig. 4. Same patients of Figs. $\mathbf{1}(\mathbf{A})$ and $\mathbf{2}(\mathbf{B})$. (A) The MR T2-weighted image demonstrates that the observation is mildly hyperintense, with an inhomogeneous structure reflecting "a mosaic architecture", and is surrounded by a hypointense distinctive rim. (B) The MR T2-weighted image shows a focal mild hyperintensity (arrow) in a larger isointense nodule, confirming the "nodule-in-nodule" architecture. 


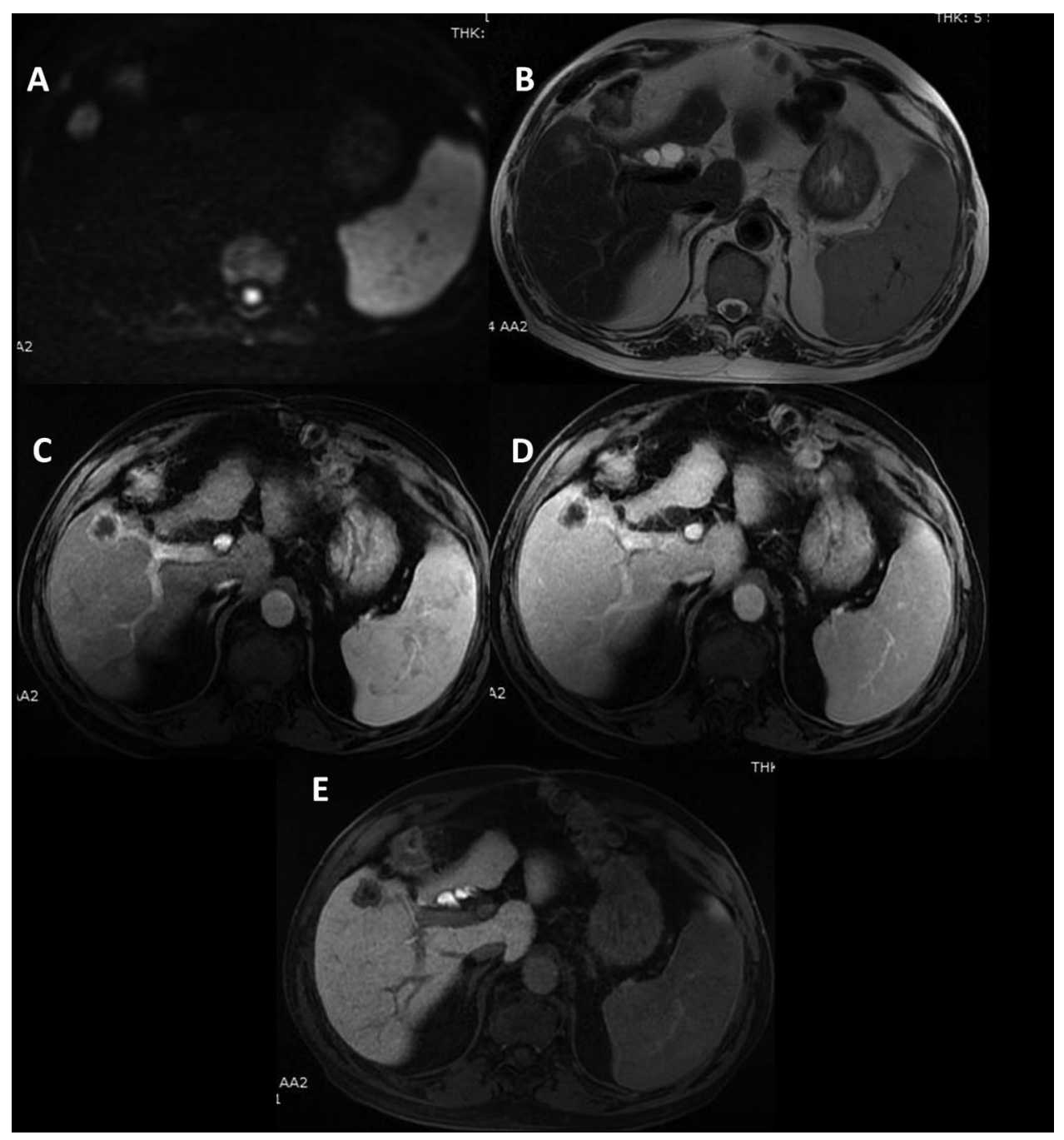

Fig. 5. A 69-year-old man with hepatitis C cirrhosis, already treated for HCC in the past, who was referred for magnetic resonance imaging after the detection of a 25-mm hypoechoic nodule in his regular ultrasound follow-up. DWI (A) and T2-weighted image (B) demonstrate the presence of a mildly hyperintense nodule, both with the characteristics of ancillary features favoring malignancy, but not HCC in particular. The dynamic phases show a targetoid appearance either in the arterial phase (note the rim hyperenhancement) (C), in the portal venous phase (D) and in the hepatobiliary phase (E), meeting the criteria for LR-M. The patient underwent a colonoscopy which found an ulcerative lesion in the left colon. A following liver biopsy confirmed that the lesion was a colon cancer metastasis.

imaging and technical parameters in order to increase sensitivity for the detection of hepatic focal lesions. ${ }^{71,74-76}$

Importantly, it must be noted that LI-RADS endorses only conventional DWI and not IntraVoxel Incoherent Motion (commonly known as IVIM) MRI, despite this method potentially enables the estimation of microvessel perfusion without the need for injection of intravenous contrast media. ${ }^{77}$ Several studies have suggested that it could have a better diagnostic performance than ADC and conventional MRI in distinguishing high-grade HCC from low-grade HCC for liver tumors diagnostic evaluation, ${ }^{78,79}$ being potentially correlated to the histological grade of tumors. ${ }^{66,69,80}$

\section{Iron sparing}

Iron sparing refers to the lack of iron deposition in a solid mass relative to an iron-overloaded liver or inside an inner nodule relative to siderotic outer nodule. ${ }^{20}$ This feature should raise concerns for premalignancy or malignancy because it is typical of high-grade dysplastic nodules and HCCs, which lose the ability to concentrate iron at the same degree of functional liver parenchyma. ${ }^{81,82}$ The LIRADS includes this feature between those favoring malignancy, but not HCC in particular, since it can be observed in other malignancies as well, such as ICCs and benign entities like confluent fibrosis. ${ }^{9}$

Iron sparing can be detected either on CT or MRI. On CT, the iron-sparing mass is less hyperdense than the background iron-overloaded liver, which is characterized by greater attenuation values than normal (usually more than $75 \mathrm{HU}$ on unenhanced images). ${ }^{9}$ On T2- or T2*-weighted images on MRI, iron-sparing observations appear as hyperintense areas compared to the hypointense siderotic hepatic parenchyma, since hepatic iron causes progressive signal loss with longer echo time, whereas HCC lesions demonstrate very little signal loss. ${ }^{83}$

The main limitation of this feature is that it can be applied only to iron-overloaded livers with solid nodules that 
unequivocally have lower fractional iron content than background parenchyma. ${ }^{9}$

\section{Corona enhancement}

LI-RADS defines corona enhancement as a periobservational enhancement in the late arterial or early portal venous phase, with fading to isoenhancement at subsequent phases. ${ }^{20}$ This feature is typical of hypervascular and progressed HCC, being correlated to the modifications of venous drainage during multistep hepatocarcinogenesis from hepatic veins to hepatic sinusoids and then to portal veins. ${ }^{84}$ Indeed, portal drainage vessels carry contrast agents from the tumor into the surrounding sinusoids, resulting in corona-shaped perilesional enhancement few seconds after the tumor itself begins to enhance. ${ }^{9}$ The corollary is that corona enhancement cannot be observed in early HCC, which are drained by hepatic veins. ${ }^{85}$

Corona enhancement is not specific for $\mathrm{HCC}$, as it may be present in hypervascular metastasis as well, ${ }^{9}$ and therefore it is included in the LI-RADS ancillary features for malignancy. ${ }^{20}$ However, it can represent a helpful tool for differentiation of small hypervascular HCCs from pseudolesions, such as arterioportal shunts, since it is always absent in the latter. ${ }^{86}$ Furthermore, evidence suggests that large or irregular and/or distorted corona enhancement predicts microvascular invasion, and therefore it can have an important prognostic value. ${ }^{87,88}$

\section{Ancillary features favoring HCC in particular}

Intralesional fat

Intralesional fat consists of excess lipid within a mass, in whole or in part, relative to the background liver. ${ }^{20}$ This feature can be best detected with MRI in association with signal loss on out-of-phase compared with in-phase T1-weighted gradient echo sequence images. Although it is relatively uncommon (having a sensitivity for HCC of $12-37 \%$ ), this feature has a high specificity for HCC (68-100\%); hence, in a high-risk population, the detection of intralesional fat in a solid hepatic nodule should raise concerns for HCC. ${ }^{23,55,89}$

Histologically, fatty metamorphosis seems to occur late during hepatocarcinogenesis, when an early-stage HCC gradually changes its blood supply from portal to arterial. ${ }^{90}$ At this transition stage, there may be some degree of ischemia, which could cause the fatty infiltration. ${ }^{90}$ However, the mechanism of this transformation is not yet fully understood..$^{90}$ It has been assessed that intralesional fat is most frequently found in early HCCs having a diameter of approximately $1.5 \mathrm{~cm}$, and that its prevalence decreases proportionally with tumor size and histologic grade. ${ }^{90}$ Furthermore, it seems that intralesional fat can have a prognostic value, since patients with fat-containing HCC may have a better clinical outcome than those without fatcontaining HCCs, due to longer progression of the tumor and decreased risk of metastasis. ${ }^{91}$

Besides HCC, fatty changes can also occur in other pathologic conditions, such as high-grade dysplastic nodule and, occasionally, in low-grade dysplastic nodules. ${ }^{86}$ Differential diagnosis also includes angioleiomyolipoma and liposarcoma, though they are unusual findings in cirrhotic livers. ${ }^{92}$ Conversely, this feature is extremely rare in ICCs and most metastases, thereby helping differential diagnosis in indeterminate cases. ${ }^{9}$ Interestingly, in steatohepatitic HCC (a newly described variant of $\mathrm{HCC}$, characterized by histologic features that resemble those of steatohepatitis in nonneoplastic liver), the fatty metamorphosis seems to appear in progressed forms with advanced tumor grade as well. ${ }^{93}$

\section{Mosaic architecture}

The LI-RADS defines mosaic architecture as the presence of randomly distributed internal nodules or compartments, usually with different imaging features (Figs. 1 and $4 \mathrm{~A}) .^{20}$ This heterogeneous appearance reflects the mosaic configuration observed at pathologic examination and is linked to the simultaneous existence inside the tumor of multiple nodules, which are featured by variable degrees of histologic differentiation, fibrous septations, necrosis, hemorrhage and occasionally copper deposition or intralesional fat. ${ }^{10,94}$ It has been demonstrated that mosaic architecture appears in $28-63 \%$ of cases of HCC, and is fairly typical of large tumors. ${ }^{95-97}$ Therefore, the utility of this ancillary sign remains uncertain in the characterization of small HCCs, but considering its high specificity for $\mathrm{HCC}$, individuation of mosaic pattern may help differentiating HCC from ICC. ${ }^{9}$

Interestingly, a recent retrospective study conducted on 275 nonmetastatic HCC larger than $5 \mathrm{~cm}$, suggested that the mosaic pattern may represent a prognostic factor as well, being associated with limited liver resection efficacy. ${ }^{94}$

\section{Nodule-in-nodule architecture}

Nodule-in-nodule architecture is defined as the presence of a smaller inner nodule within a larger outer nodule, with different imaging features (Figs. 2C, 2D and 4B). ${ }^{20}$ Histologically, such a condition corresponds to a developing focus of localized, progressed $\mathrm{HCC}^{98}$ within a well-differentiated $\mathrm{HCC}$ or dysplastic nodule, occasionally still rich in fat or iron. ${ }^{9,10}$ Thus, a nodule-in-nodule appearance may be interpreted as a morphologic marker of the progression of dedifferentiation of the tumor. ${ }^{99}$ By MRI observation, the inner nodule may show other major or ancillary features for HCC, while the surrounding nodule demonstrates features of benignity. ${ }^{100}$ The presence of nodule-in-nodule appearance of HCC as a prognostic indicator is not established. ${ }^{9}$

\section{Diagnostic algorithm}

In this section, we will focus on untreated observations.

When a radiologist approaches a CT or MRI liver examination, the first thing he/she is supposed to do is to verify whether the available images are technically adequate for LI-RADS application. If the quality of the exam is not sufficient to allow an interpretation, the observation should be defined as LR-noncategorizable. ${ }^{20}$ In the opposite case, the radiologist can apply the LI-RADS algorithm, providing a categorization for each observation detected in the liver. Indeed, it should be noted that the LI-RADS does not assign a category to the entire liver, but it contemplates the coexistence in the same organ of multiple observations that can be classified differently. ${ }^{18}$

The first step is to verify whether the observation meets the criteria of benignity. The LI-RADS defines benign entities as solid nodules with a distinctive imaging appearance compared to background cirrhotic nodules, with no major features of HCC or any other ancillary features of malignancy. Namely, a benign nodule is supposed to be characterized by T1 hyperintensity, low or very high T2 signal, HBP hyper/isointensity, marked iron 
Table 4. LI-RADS tie-breaking rules for uncertain situations. From the American College of Radiology - Liver imaging reporting and data system version (LI-RADS $®)$ V 2017
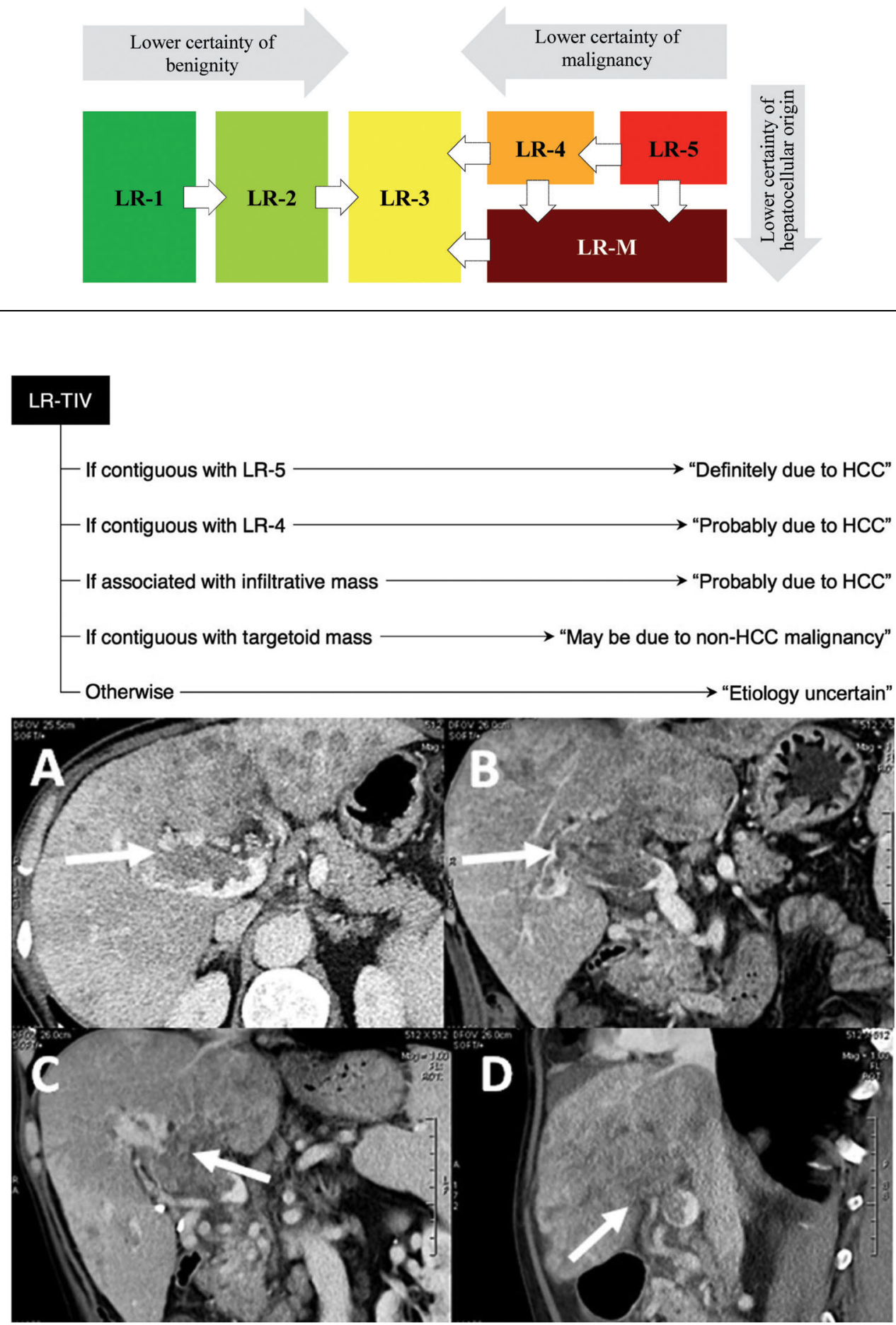

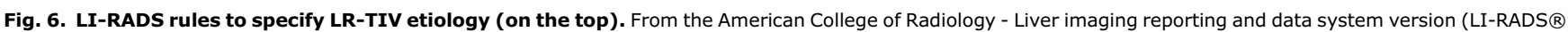
v2017. Cirrhotic 56-year-old patient (on the bottom). The axial (A), the coronal (B, C) and the sagittal (D) computed tomography images show an infiltrating lesion of the left lobe invading the portal vein at the bifurcation, with involvement of both the left and right portal branches. The observation was definitively a LR-TIV. Since the contiguous lesion was $>2 \mathrm{~cm}$, presented a "wash-out" (major criteria), but not a clear APHE in the arterial phase (not in this box), it could be classified as an LR-4. Thus, the correct definition in reports should be LR-TIV probably due to HCC. The lesion was biopsied and resulted in a diagnosis of HCC. The patient underwent sorafenib therapy. 
concentration or any combination of them. ${ }^{20}$ Moreover, an observation can be classified as benign only if its size does not increase and if its diameter does not exceed $20 \mathrm{~mm}$. Importantly, if the diameter is more than $20 \mathrm{~mm}$, the observation should be categorized as LR-3. ${ }^{20}$ If the imaging features allows a certain diagnosis of benignity, the observation should be labelled as LR-1; otherwise, if the probability of benignity is still high but less than $100 \%$, the observation should be classified as LR-2. Examples of LR-1 and LR-2 entities are listed in Table 2.

Interestingly, when the findings are suggestive of FNH or HCA, the LI-RADS recommends to categorize the observation as LR-3 or, with caution, as LR-2, but never as LR-1. The rationale is that these are supposed to be diagnoses of exclusion in high-risk patients. ${ }^{20}$ Whether the observation does not meet the criteria for LR-1 or LR-2, the subsequent step is to look for the presence of major criteria for HCC. Namely, the radiologist should assess if an APHE, with the fore-mentioned features (i.e. not rim enhancement) can be detected. This should be the first step since, as it is shown in Fig. 1, the LI-RADS algorithm uses the presence or the absence of APHE to delineate two different situations: observations without $A P H E$, which should be classified employing the guidelines in the left part of the table; and, observations with $A P H E$, which need to be categorized using the right part of the table. ${ }^{20}$ Thus, according to the size and the presence of other major features, lesions without APHE can be labelled just as LR-3 or LR-4, while those with APHE can range from LR-3 to LR-5. ${ }^{20}$

Indeed, unlike other RADS systems, the LI-RADS algorithm was conceived to define, by application of stringent diagnostic criteria, a LR-5 category, featuring nearly $100 \%$ specificity of $\mathrm{HCC}$ and eliminating the need for biopsy. ${ }^{19}$ Therefore, observations without $A P H E$, regardless of their size, and observations less than $10 \mathrm{~mm}$, despite their imaging features, cannot be classified as LR-5, which is consistent with the United Network for Organ Sharing requirements. ${ }^{19}$ Furthermore, LI-RADS recommends the application of major criteria only if they can be unequivocally detected. ${ }^{20}$ Given these considerations, clinicians should be conscious that a LI-RADS category less than LR-5 does not exclude HCC. ${ }^{18}$

Interestingly, the right side of the table includes a "split" cell, corresponding to observations with APHE, sizing of 10-19 mm, and one other major feature. These observations can be up-graded to LR-5 only if they were reported as a distinctive nodule on a former ultrasound examination, or they achieve the threshold growth of $\geq 50 \%$ in $<6$ months; in the first case, the lesions should be assigned to the category LR-5us, while in second one they should be depicted as LR-5g. In all the other instances, the observation must be classified as LR-4. ${ }^{19,20}$

Once the evaluation of the major features is concluded and an initial category is assigned, the radiologist can decide, according to her/his discretion, whether to apply the ancillary features to adjust the label, especially if she/he disposes of a multiparametric MRI examination. The LI-RADS v2017 provides few rules for their employment (Fig. 3). ${ }^{20}$ First of all, ancillary features do not upgrade LR-4 to the LR-5 category. In addition, the use of ancillary features cannot allow a change of category by more than one level, regardless of the number of features. Eventually, if there are conflicting ancillary features (some of them favoring benignity and some of them favoring malignancy), the label should not be adjusted. ${ }^{20}$
The LI-RADS also provides guidelines, called "tie-breaking rules", to overcome the cases of indecision between two categories generally, recommending the choice of the alternative which involves the higher degree of uncertainty (Table 4). ${ }^{20}$ Figs. 1-3 present some examples of LR-4 and LR-5.

When the findings suggest malignancy, but they are not specific for HCC, the advisable category should be LRm. Namely, this category should include all nodular alterations featured by a targetoid appearance (concerning either the dynamic pattern or the morphological aspect in DWI or HBP), infiltrative appearance, marked diffusion restriction, necrosis or severe ischemia or other feature that in the radiologist's judgment suggests a nonHCC malignancy (Fig. 5). ${ }^{20}$ Once the malignancy is assessed (LR-5 or LRm) or a HCC is highly suspected (LR-4), the radiologist is supposed to verify the portal involvement by the tumor. When an unequivocal enhancing soft tissue is present in the vein, the report should be categorized as LR-TIV and the etiology must be indicated as shown in Fig. 6. Importantly, the assignment of this category does not necessarily require the visualization of a parenchymal mass. ${ }^{20}$

\section{Conclusions}

The LI-RADS provides a diagnostic algorithm aimed at standardizing the interpretations and reports of liver observations in a high-risk population for $\mathrm{HCC}$, in order to optimize patient management. This system was conceived to reduce the indeterminacy, classifying the observations in five categories corresponding to increasing probability of HCC on the basis of the unequivocal presence of major and ancillary features. Furthermore, different from other organs Reporting and Data Systems, the LI-RADS enables the diagnosis of HCC without the need for any further biopsy, designing a LR-5 category featuring $100 \%$ specificity. Thus, radiologists and clinicians must be aware that not-benign categories less than LR-5 do not exclude the diagnosis of HCC, and that the LR-4 observation namely, just like the LR-5, may deserve a multidisciplinary discussion for consensus management. ${ }^{20}$

\section{Conflict of interest}

The authors have no conflict of interests related to this publication.

\section{Author contributions}

Conception and design (FrP, FiP, EMF), administrative support (FrP, FiP, GF, GC), provision of study materials or patients, collection and assembly of data, data analysis and interpretation, manuscript writing and final approval of manuscript for publication (FrP, FiP, EMF, IE, RS, SAA, ST, GF, GC).

\section{References}

[1] Ferlay J, Soerjomataram I, Dikshit R, Eser S, Mathers C, Rebelo M, et al. Cancer incidence and mortality worldwide: sources, methods and major patterns in GLOBOCAN 2012. Int J Cancer 2015;136:E359-E386. doi: 10. 1002/ijc. 29210.

[2] McGlynn KA, London WT. Epidemiology and natural history of hepatocellular carcinoma. Best Pract Res Clin Gastroenterol 2005;19:3-23. doi: 10.1016/ j.bpg.2004.10.004.

[3] Kudo M. Real practice of hepatocellular carcinoma in Japan: conclusions of the Japan Society of Hepatology 2009 Kobe Congress. Oncology 2010;78: 180-188. doi: 10.1159/000315740. 
[4] El-Serag HB. Hepatocellular carcinoma. N Engl J Med 2011;365:1118-1127. doi: 10.1056/NEJMra1001683.

[5] Baffy G, Brunt EM, Caldwell SH. Hepatocellular carcinoma in non-alcoholic fatty liver disease: an emerging menace. J Hepatol 2012;56:1384-1391. doi: 10.1016/j.jhep.2011.10.027.

[6] Tyson GL, El-Serag HB. Risk factors for cholangiocarcinoma. Hepatology 2011;54:173-184. doi: 10.1002/hep.24351.

[7] Ierardi AM, Angileri SA, Patella F, Panella S, Lucchina N, Petre EN, et al. The role of interventional radiology in the treatment of intrahepatic cholangiocarcinoma. Med Oncol 2017;34:11. doi: 10.1007/s12032-016-0866-1.

[8] Dahl E, Rumessen J, Gluud LL. Systematic review with meta-analyses of studies on the association between cirrhosis and liver metastases. Hepatol Res 2011;41:618-625. doi: 10.1111/j.1872-034X.2011.00813.x.

[9] Choi JY, Lee JM, Sirlin CB. CT and MR imaging diagnosis and staging of hepatocellular carcinoma: part II. Extracellular agents, hepatobiliary agents, and ancillary imaging features. Radiology 2014;273:30-50. doi: 10.1148/radiol.14132362.

[10] Choi JY, Lee JM, Sirlin CB. CT and MR imaging diagnosis and staging of hepatocellular carcinoma: part I. Development, growth, and spread: key pathologic and imaging aspects. Radiology 2014;272:635-654. doi: 10. 1148/radiol.14132361.

[11] Pesapane F, Nezami N, Patella F, Geschwind JF. New concepts in embolotherapy of HCC. Med Oncol 2017;34:58. doi: 10.1007/s12032-017-0917-2.

[12] de Lope CR, Tremosini S, Forner A, Reig M, Bruix J. Management of HCC. J Hepatol 2012;56:S75-S87. doi: 10.1016/S0168-8278(12)60009-9.

[13] Bruix J, Sherman M. Management of hepatocellular carcinoma: an update. Hepatology 2011;53:1020-1022. doi: 10.1002/hep.24199.

[14] EASL-EORTC clinical practice guidelines: management of hepatocellular carcinoma. J Hepatol 2012;56:908-943. doi: 10.1016/j.jhep.2011.12.001.

[15] Aubé C, Oberti F, Lonjon J, Pageaux G, Seror O, N'Kontchou G, et al. EASL and AASLD recommendations for the diagnosis of HCC to the test of daily practice. Liver Int 2017;37:1515-1525. doi: 10.1111/liv.13429.

[16] Cruite I, Tang A, Sirlin CB. Imaging-based diagnostic systems for hepatocellular carcinoma. AJR Am J Roentgenol 2013;201:41-55. doi: 10.2214/ AJR.13.10570.

[17] Granata V, Fusco R, Avallone A, Catalano O, Filice F, Leongito M, et al. Major and ancillary magnetic resonance features of LI-RADS to assess HCC: an overview and update. Infect Agent Cancer 2017;12:23. doi: 10.1186/ s13027-017-0132-y.

[18] Santillan CS, Tang A, Cruite I, Shah A, Sirlin CB. Understanding LI-RADS: a primer for practical use. Magn Reson Imaging Clin N Am 2014;22:337-352. doi: 10.1016/j.mric.2014.04.007.

[19] Chernyak V, Santillan CS, Papadatos D, Sirlin CB. LI-RADS $®$ algorithm: CT and MRI. Abdom Radiol (NY) 2018;43:111-126. doi: 10.1007/s00261017-1228-y.

[20] CT/MRI LI-RADS $®$ v2017. Available from: https://www.acr.org/ClinicalResources/Reporting-and-Data-Systems/LI-RADS/CT-MRI-LI-RADS-v2017.

[21] Forner A, Vilana R, Ayuso C, Bianchi L, Solé M, Ayuso JR, et al. Diagnosis of hepatic nodules $20 \mathrm{~mm}$ or smaller in cirrhosis: Prospective validation of the noninvasive diagnostic criteria for hepatocellular carcinoma. Hepatology 2008;47:97-104. doi: 10.1002/hep.21966.

[22] Kim TK, Lee KH, Jang HJ, Haider MA, Jacks LM, Menezes RJ, et al. Analysis of gadobenate dimeglumine-enhanced MR findings for characterizing small (1-2-cm) hepatic nodules in patients at high risk for hepatocellular carcinoma. Radiology 2011;259:730-738. doi: 10.1148/radiol.11101549.

[23] Rimola J, Forner A, Tremosini S, Reig M, Vilana R, Bianchi L, et al. Noninvasive diagnosis of hepatocellular carcinoma $\leq 2 \mathrm{~cm}$ in cirrhosis. Diagnostic accuracy assessing fat, capsule and signal intensity at dynamic MRI. J Hepatol 2012;56:1317-1323. doi: 10.1016/j.jhep.2012.01.004.

[24] Efremidis SC, Hytiroglou P. The multistep process of hepatocarcinogenesis in cirrhosis with imaging correlation. Eur Radiol 2002;12:753-764. doi: 10. 1007/s00330-001-1142-z.

[25] Lee JM, Choi BI. Hepatocellular nodules in liver cirrhosis: MR evaluation. Abdom Imaging 2011;36:282-289. doi: 10.1007/s00261-011-9692-2.

[26] Sano K, Ichikawa T, Motosugi U, Sou H, Muhi AM, Matsuda M, et al. Imaging study of early hepatocellular carcinoma: usefulness of gadoxetic acidenhanced MR imaging. Radiology 2011;261:834-844. doi: 10.1148/radiol. 11101840.

[27] Holland AE, Hecht EM, Hahn WY, Kim DC, Babb JS, Lee VS, et al. Importance of small $(<$ or $=20-\mathrm{mm}$ ) enhancing lesions seen only during the hepatic arterial phase at MR imaging of the cirrhotic liver: evaluation and comparison with whole explanted liver. Radiology 2005;237:938-944. doi: 10. 1148/radiol.2373041364.

[28] Hwang SH, Yu JS, Kim KW, Kim JH, Chung JJ. Small hypervascular enhancing lesions on arterial phase images of multiphase dynamic computed tomography in cirrhotic liver: fate and implications. J Comput Assist Tomogr 2008;32:39-45. doi: 10.1097/RCT.0b013e318064c76b.

[29] Burrel M, Llovet JM, Ayuso C, Iglesias C, Sala M, Miquel R, et al. MRI angiography is superior to helical CT for detection of HCC prior to liver transplantation: an explant correlation. Hepatology 2003;38:1034-1042. doi: 10.1053/jhep.2003.50409.

[30] Lim JH, Choi D, Kim SH, Lee SJ, Lee WJ, Lim HK, et al. Detection of hepatocellular carcinoma: value of adding delayed phase imaging to dual-phase helical CT. AJR Am J Roentgenol 2002;179:67-73. doi: 10.2214/ajr.179.1. 1790067.

[31] Monzawa S, Ichikawa T, Nakajima H, Kitanaka Y, Omata K, Araki T. Dynamic CT for detecting small hepatocellular carcinoma: usefulness of delayed phase imaging. AJR Am J Roentgenol 2007;188:147-153. doi: 10.2214/ AJR.05.0512.

[32] Marrero JA, Hussain HK, Nghiem HV, Umar R, Fontana RJ, Lok AS. Improving the prediction of hepatocellular carcinoma in cirrhotic patients with an arterially-enhancing liver mass. Liver Transpl 2005;11:281-289. doi: 10. $1002 /$ lt.20357.

[33] Calderaro J, Nault JC, Balabaud C, Couchy G, Saint-Paul MC, Azoulay D, et al. Inflammatory hepatocellular adenomas developed in the setting of chronic liver disease and cirrhosis. Mod Pathol 2016;29:43-50. doi: 10. 1038/modpathol.2015.119.

[34] Khan AS, Hussain HK, Johnson TD, Weadock WJ, Pelletier SJ, Marrero JA. Value of delayed hypointensity and delayed enhancing rim in magnetic resonance imaging diagnosis of small hepatocellular carcinoma in the cirrhotic liver. J Magn Reson Imaging 2010;32:360-366. doi: 10.1002/jmri.22271.

[35] Grazioli L, Olivetti L, Fugazzola C, Benetti A, Stanga C, Dettori E, et al. The pseudocapsule in hepatocellular carcinoma: correlation between dynamic MR imaging and pathology. Eur Radiol 1999;9:62-67. doi: 10.1007/ s003300050629.

[36] Ishigami $\mathrm{K}$, Yoshimitsu $\mathrm{K}$, Nishihara $\mathrm{Y}$, Irie $\mathrm{H}$, Asayama $\mathrm{Y}$, Tajima $\mathrm{T}$, et al. Hepatocellular carcinoma with a pseudocapsule on gadolinium-enhanced MR images: correlation with histopathologic findings. Radiology 2009; 250:435-443. doi: 10.1148/radiol.2501071702.

[37] Lim JH, Choi D, Park CK, Lee W], Lim HK. Encapsulated hepatocellular carcinoma: CT-pathologic correlations. Eur Radiol 2006;16:2326-2333. doi: 10.1007/s00330-006-0203-8.

[38] Pomfret EA, Washburn K, Wald C, Nalesnik MA, Douglas D, Russo M, et al. Report of a national conference on liver allocation in patients with hepatocellular carcinoma in the United States. Liver Transpl 2010;16:262-278. doi: $10.1002 /$ It.21999.

[39] Rimola J, Forner A, Reig M, Vilana R, de Lope CR, Ayuso C, et al. Cholangiocarcinoma in cirrhosis: absence of contrast washout in delayed phases by magnetic resonance imaging avoids misdiagnosis of hepatocellular carcinoma. Hepatology 2009;50:791-798. doi: 10.1002/hep.23071.

[40] Yu JS, Lee JH, Chung J], Kim JH, Kim KW. Small hypervascular hepatocellular carcinoma: limited value of portal and delayed phases on dynamic magnetic resonance imaging. Acta Radiol 2008;49:735-743. doi: 10. 1080/02841850802120045.

[41] Hennedige T, Venkatesh SK. Advances in computed tomography and magnetic resonance imaging of hepatocellular carcinoma. World J Gastroenterol 2016;22:205-220. doi: 10.3748/wjg.v22.i1.205.

[42] Gatto A, De Gaetano AM, Giuga M, Ciresa M, Siciliani L, Miele L, et al. Differentiating hepatocellular carcinoma from dysplastic nodules at gadobenate dimeglumine-enhanced hepatobiliary-phase magnetic resonance imaging. Abdom Imaging 2013;38:736-744. doi: 10.1007/s00261-012-9950-y.

[43] Pahade JK, Juice D, Staib L, Israel G, Cornfeld D, Mitchell K, et al. Is there an added value of a hepatobiliary phase with gadoxetate disodium following conventional MRI with an extracellular gadolinium agent in a single imaging session for detection of primary hepatic malignancies? Abdom Radiol (NY) 2016;41:1270-1284. doi: 10.1007/s00261-016-0635-9.

[44] Motosugi U, Bannas P, Sano K, Reeder SB. Hepatobiliary MR contrast agents in hypovascular hepatocellular carcinoma. J Magn Reson Imaging 2015;41: 251-265. doi: $10.1002 /$ jmri.24712.

[45] Hope TA, Fowler KJ, Sirlin CB, Costa EA, Yee J, Yeh BM, et al. Hepatobiliary agents and their role in LI-RADS. Abdom Imaging 2015;40:613-625. doi: 10.1007/s00261-014-0227-5.

[46] Chernyak V, Tang A, Flusberg M, Papadatos D, Bijan B, Kono Y, et al. LI-RADS $®$ ancillary features on CT and MRI. Abdom Radiol (NY) 2018;43:82-100. doi: 10.1007/s00261-017-1220-6.

[47] Rhee H, Kim MJ, Park YN, Choi JS, Kim KS. Gadoxetic acid-enhanced MRI findings of early hepatocellular carcinoma as defined by new histologic criteria. J Magn Reson Imaging 2012;35:393-398. doi: 10.1002/jmri.22828.

[48] Nowicki TK, Markiet K, Szurowska E. Diagnostic imaging of hepatocellular carcinoma - A pictorial essay. Curr Med Imaging Rev 2017;13:140-153. doi: $10.2174 / 1573405612666160720123748$

[49] Ronot M, Dioguardi Burgio M, Purcell Y, Pommier R, Brancatelli G, Vilgrain V. Focal lesions in cirrhosis: Not always HCC. Eur J Radiol 2017;93:157-168. doi: 10.1016/j.ejrad.2017.05.040.

[50] Kadoya M, Matsui O, Takashima T, Nonomura A. Hepatocellular carcinoma: correlation of MR imaging and histopathologic findings. Radiology 1992 183:819-825. doi: 10.1148/radiology.183.3.1316622.

[51] Shinmura R, Matsui O, Kobayashi S, Terayama N, Sanada J, Ueda K, et al. Cirrhotic nodules: association between MR imaging signal intensity and 
intranodular blood supply. Radiology 2005;237:512-519. doi: 10.1148/ radiol.2372041389.

[52] Choi MH, Choi JI, Lee YJ, Park MY, Rha SE, Lall C. MRI of small hepatocellular carcinoma: typical features are less frequent below a size cutoff of $1.5 \mathrm{~cm}$. AJR Am J Roentgenol 2017;208:544-551. doi: 10.2214/AJR.16.16414.

[53] Iwasa Y, Kitazume Y, Tateishi U, Saida Y, Ban D, Tanabe M, et al. Hepatocellular carcinoma histological grade prediction: a quantitative comparison of diffusion-weighted, T2-weighted, and hepatobiliary-phase magnetic resonance imaging. J Comput Assist Tomogr 2016;40:463-470. doi: 10.1097/ RCT.0000000000000367.

[54] Chou CT, Chou JM, Chang TA, Huang SF, Chen CB, Chen YL, et al. Differentiation between dysplastic nodule and early-stage hepatocellular carcinoma: the utility of conventional MR imaging. World J Gastroenterol 2013; 19:7433-7439. doi: 10.3748/wjg.v19.i42.7433.

[55] Ramalho M, Matos AP, AlObaidy M, Velloni F, Altun E, Semelka RC. Magnetic resonance imaging of the cirrhotic liver: diagnosis of hepatocellular carcinoma and evaluation of response to treatment - Part 2. Radiol Bras 2017; 50:115-125. doi: 10.1590/0100-3984.2015.0140.

[56] Shin SK, Kim YS, Choi SJ, Shim YS, Jung DH, Kwon OS, et al. Characterization of small $(\leq 3 \mathrm{~cm})$ hepatic lesions with atypical enhancement feature and hypointensity in hepatobiliary phase of gadoxetic acid-enhanced MRI in cirrhosis: A STARD-compliant article. Medicine (Baltimore) 2017;96:e7278. doi: 10.1097/MD.0000000000007278.

[57] Merkle EM, Zech C], Bartolozzi C, Bashir MR, Ba-Ssalamah A, Huppertz A, et al. Consensus report from the 7th International Forum for Liver Magnetic Resonance Imaging. Eur Radiol 2016;26:674-682. doi: 10.1007/s00330015-3873-2.

[58] Le Bihan D, Breton E, Lallemand D, Grenier P, Cabanis E, Laval-Jeantet M. MR imaging of intravoxel incoherent motions: application to diffusion and perfusion in neurologic disorders. Radiology 1986;161:401-407. doi: 10. 1148/radiology.161.2.3763909.

[59] Li YT, Cercueil JP, Yuan J, Chen W, Loffroy R, Wáng YX. Liver intravoxel incoherent motion (IVIM) magnetic resonance imaging: a comprehensive review of published data on normal values and applications for fibrosis and tumor evaluation. Quant Imaging Med Surg 2017;7:59-78. doi: 10.21037/ qims.2017.02.03.

[60] Bruegel M, Gaa J, Waldt S, Woertler K, Holzapfel K, Kiefer B, et al. Diagnosis of hepatic metastasis: comparison of respiration-triggered diffusionweighted echo-planar MRI and five t2-weighted turbo spin-echo sequences. AJR Am J Roentgenol 2008;191:1421-1429. doi: 10.2214/AJR.07. 3279.

[61] Coenegrachts K, Delanote J, Ter Beek L, Haspeslagh M, Bipat S, Stoker J, et al. Improved focal liver lesion detection: comparison of single-shot diffusion-weighted echoplanar and single-shot T2 weighted turbo spin echo techniques. $\mathrm{Br}$ J Radiol 2007;80:524-531. doi: 10.1259/bjr/ 33156643.

[62] Zech C], Reiser MF, Herrmann KA. Imaging of hepatocellular carcinoma by computed tomography and magnetic resonance imaging: state of the art. Dig Dis 2009;27:114-124. doi: 10.1159/000218343.

[63] Vandecaveye V, De Keyzer F, Verslype C, Op de Beeck K, Komuta M, Topal B, et al. Diffusion-weighted MRI provides additional value to conventional dynamic contrast-enhanced MRI for detection of hepatocellular carcinoma. Eur Radiol 2009;19:2456-2466. doi: 10.1007/s00330-009-1431-5.

[64] Kele PG, van der Jagt EJ. Diffusion weighted imaging in the liver. World J Gastroenterol 2010;16:1567-1576. doi: 10.3748/wjg.v16.i13.1567.

[65] Granata V, Fusco R, Catalano O, Guarino B, Granata F, Tatangelo F, et al. Intravoxel incoherent motion (IVIM) in diffusion-weighted imaging (DWI) for Hepatocellular carcinoma: correlation with histologic grade. Oncotarget 2016;7:79357-79364. doi: 10.18632/oncotarget.12689.

[66] Woo S, Lee JM, Yoon JH, Joo I, Han JK, Choi BI. Intravoxel incoherent motion diffusion-weighted MR imaging of hepatocellular carcinoma: correlation with enhancement degree and histologic grade. Radiology 2014;270: 758-767. doi: 10.1148/radiol.13130444.

[67] Nishie A, Tajima T, Asayama Y, Ishigami K, Kakihara D, Nakayama T, et al. Diagnostic performance of apparent diffusion coefficient for predicting histological grade of hepatocellular carcinoma. Eur J Radiol 2011;80: e29-e33. doi: 10.1016/j.ejrad.2010.06.019.

[68] Heo SH, Jeong YY, Shin SS, Kim JW, Lim HS, Lee JH, et al. Apparent diffusion coefficient value of diffusion-weighted imaging for hepatocellular carcinoma: correlation with the histologic differentiation and the expression of vascular endothelial growth factor. Korean J Radiol 2010;11:295-303. doi: 10.3348/kjr.2010.11.3.295

[69] Guo W, Zhao S, Yang Y, Shao G. Histological grade of hepatocellular carcinoma predicted by quantitative diffusion-weighted imaging. Int J Clin Exp Med 2015;8:4164-4169.

[70] Yamada I, Aung W, Himeno Y, Nakagawa T, Shibuya H. Diffusion coefficients in abdominal organs and hepatic lesions: evaluation with intravoxel incoherent motion echo-planar MR imaging. Radiology 1999;210:617-623. doi: 10.1148/radiology.210.3.r99fe17617.
[71] Iima M, Le Bihan D. Clinical intravoxel incoherent motion and diffusion MR imaging: past, present, and future. Radiology 2016;278:13-32. doi: 10 . 1148/radiol.2015150244.

[72] Kwee TC, Takahara T, Koh DM, Nievelstein RA, Luijten PR. Comparison and reproducibility of $A D C$ measurements in breathhold, respiratory triggered, and free-breathing diffusion-weighted MR imaging of the liver. J Magn Reson Imaging 2008;28:1141-1148. doi: 10.1002/jmri.21569.

[73] Liau J, Lee J, Schroeder ME, Sirlin CB, Bydder M. Cardiac motion in diffusionweighted MRI of the liver: artifact and a method of correction. J Magn Reson Imaging 2012;35:318-327. doi: 10.1002/jmri.22816.

[74] Kim YK, Lee MW, Lee WJ, Kim SH, Rhim H, Lim JH, et al. Diagnostic accuracy and sensitivity of diffusion-weighted and of gadoxetic acid-enhanced 3-T MR imaging alone or in combination in the detection of small liver metastasis ( $\leq 1.5 \mathrm{~cm}$ in diameter). Invest Radiol 2012;47:159-166. doi: 10 . 1097/RLI.0b013e31823a1495.

[75] Holzapfel K, Eiber MJ, Fingerle AA, Bruegel M, Rummeny EJ, Gaa J. Detection, classification, and characterization of focal liver lesions: Value of diffusion-weighted MR imaging, gadoxetic acid-enhanced MR imaging and the combination of both methods. Abdom Imaging 2012;37:74-82. doi: 10.1007/s00261-011-9758-1.

[76] Chung WS, Kim MJ, Chung YE, Kim YE, Park MS, Choi JY, et al. Comparison of gadoxetic acid-enhanced dynamic imaging and diffusion-weighted imaging for the preoperative evaluation of colorectal liver metastases. J Magn Reson Imaging 2011;34:345-353. doi: 10.1002/jmri.22671.

[77] Le Bihan D, Breton E, Lallemand D, Aubin ML, Vignaud J, Laval-Jeantet M. Separation of diffusion and perfusion in intravoxel incoherent motion MR imaging. Radiology 1988;168:497-505. doi: 10.1148/radiology.168.2. 3393671.

[78] Yoon JH, Lee JM, Yu MH, Kiefer B, Han JK, Choi BI. Evaluation of hepatic focal lesions using diffusion-weighted MR imaging: comparison of apparent diffusion coefficient and intravoxel incoherent motion-derived parameters. J Magn Reson Imaging 2014;39:276-285. doi: 10.1002/jmri.24158.

[79] Nakanishi M, Chuma M, Hige S, Omatsu T, Yokoo H, Nakanishi K, et al. Relationship between diffusion-weighted magnetic resonance imaging and histological tumor grading of hepatocellular carcinoma. Ann Surg Oncol 2012;19:1302-1309. doi: 10.1245/s10434-011-2066-8.

[80] Pesapane F, Patella F, Fumarola EM, Panella S, Ierardi AM, Pompili GG, et al. Intravoxel incoherent motion (IVIM) diffusion weighted imaging (DWI) in the periferic prostate cancer detection and stratification. Med Oncol 2017; 34:35. doi: 10.1007/s12032-017-0892-7.

[81] Zhang J, Krinsky GA. Iron-containing nodules of cirrhosis. NMR Biomed 2004;17:459-464. doi: 10.1002/nbm.926.

[82] Pomerantz S, Siegelman ES. MR imaging of iron depositional disease. Magn Reson Imaging Clin N Am 2002;10:105-120. doi: 10.1016/S1064-9689 (03)00052-7.

[83] Hardie AD, Nance JW, Boulter D], Kizziah MK. Assessment of the diagnostic accuracy of $\mathrm{T} 2 *$-weighted MR imaging for identifying hepatocellular carcinoma with liver explant correlation. Eur J Radiol 2011;80:e249-e252. doi: 10.1016/j.ejrad.2010.10.027.

[84] Kitao A, Zen Y, Matsui O, Gabata T, Nakanuma Y. Hepatocarcinogenesis: multistep changes of drainage vessels at CT during arterial portography and hepatic arteriography-radiologic-pathologic correlation. Radiology 2009;252: 605-614. doi: 10.1148/radiol.2522081414.

[85] Ito K, Fujita T, Shimizu A, Koike S, Sasaki K, Matsunaga N, et al. Multiarterial phase dynamic MRI of small early enhancing hepatic lesions in cirrhosis or chronic hepatitis: differentiating between hypervascular hepatocellular carcinomas and pseudolesions. AJR Am J Roentgenol 2004;183:699-705. doi: 10.2214/ajr.183.3.1830699.

[86] Kanematsu M, Kondo H, Goshima S, Tsuge Y, Watanabe H. Magnetic resonance imaging of hepatocellular carcinoma. Oncology 2008;75:65-71. doi: $10.1159 / 000173426$.

[87] Nishie A, Yoshimitsu K, Asayama Y, Irie H, Tajima T, Hirakawa M, et al. Radiologic detectability of minute portal venous invasion in hepatocellular carcinoma. AJR Am J Roentgenol 2008;190:81-87. doi: 10.2214/AJR.07.2810.

[88] Kim H, Park MS, Choi JY, Park YN, Kim MJ, Kim KS, et al. Can microvessel invasion of hepatocellular carcinoma be predicted by pre-operative MRI? Eur Radiol 2009;19:1744-1751. doi: 10.1007/s00330-009-1331-8.

[89] Yu JS, Chung J], Kim JH, Kim KW. Fat-containing nodules in the cirrhotic liver: chemical shift MRI features and clinical implications. AJR Am J Roentgenol 2007;188:1009-1016. doi: 10.2214/AJR.06.0756.

[90] Kutami R, Nakashima Y, Nakashima O, Shiota K, Kojiro M. Pathomorphologic study on the mechanism of fatty change in small hepatocellular carcinoma of humans. J Hepatol 2000;33:282-289. doi: 10.1016/S0168-8278(00) 80369-4.

[91] Siripongsakun S, Lee JK, Raman SS, Tong MJ, Sayre J, Lu DS. MRI detection of intratumoral fat in hepatocellular carcinoma: potential biomarker for a more favorable prognosis. AJR Am J Roentgenol 2012;199:1018-1025. doi: $10.2214 /$ AJR.12.8632.

[92] Sun H, Song T. Hepatocellular carcinoma: Advances in diagnostic imaging. Drug Discov Ther 2015;9:310-318. doi: 10.5582/ddt.2015.01058. 
[93] Salomao M, Remotti $H$, Vaughan $R$, Siegel AB, Lefkowitch JH, Moreira RK. The steatohepatitic variant of hepatocellular carcinoma and its association with underlying steatohepatitis. Hum Pathol 2012;43:737-746. doi: 10. 1016/j.humpath.2011.07.005.

[94] Li M, Xin Y, Fu S, Liu Z, Li Y, Hu B, et al. Corona enhancement and mosaic architecture for prognosis and selection between of liver resection versus transcatheter arterial chemoembolization in single hepatocellular carcinomas $>5 \mathrm{~cm}$ without extrahepatic metastases: an imaging-based retrospective study. Medicine (Baltimore) 2016;95:e2458. doi: 10.1097/ MD.0000000000002458.

[95] Samanci C, Sobhani F, Ucbilek E, Rakestraw K, Dagher NN, Kamel IR, et al. Magnetic resonance imaging in diagnosis and monitoring of hepatocellular carcinoma in liver transplantation: a comprehensive review. Ann Transplant 2016;21:68-76. doi: 10.12659/AOT.895595.

[96] Stevens WR, Gulino SP, Batts KP, Stephens DH, Johnson CD. Mosaic pattern of hepatocellular carcinoma: histologic basis for a characteristic
CT appearance. J Comput Assist Tomogr 1996;20:337-342. doi: 10.1097/ 00004728-199605000-00001.

[97] van den Bos IC, Hussain SM, Dwarkasing RS, Hop WC, Zondervan PE, de Man RA, et al. MR imaging of hepatocellular carcinoma: relationship between lesion size and imaging findings, including signal intensity and dynamic enhancement patterns. J Magn Reson Imaging 2007;26:15481555. doi: 10.1002/jmri.21046.

[98] Sadek AG, Mitchell DG, Siegelman ES, Outwater EK, Matteuccí T, Hann HW. Early hepatocellular carcinoma that develops within macroregenerative nodules: growth rate depicted at serial MR imaging. Radiology 1995;195: 753-756. doi: 10.1148/radiology.195.3.7754006.

[99] Kojiro M. 'Nodule-in-nodule' appearance in hepatocellular carcinoma: its significance as a morphologic marker of dedifferentiation. Intervirology 2004;47:179-183. doi: 10.1159/000078470.

[100] Cho ES, Choi JY. MRI features of hepatocellular carcinoma related to biologic behavior. Korean J Radiol 2015;16:449-464. doi: 10.3348/kjr.2015.16.3.449. 